\title{
Water level collapse effect of deep well in mining area
}

\author{
LIN Mei*, DONG Xiaona \\ Shandong Earthquake Agency, Jinan 250014, Shandong, China
}

\begin{abstract}
The use of Zaozhuang of Shandong province coal mine area is tao chong lu 15 Well and meteorological observation data, the three elements of observation Wells with tao chong effect comparing the dynamic characteristics of the mines. It is difficult to determine whether the stress state of the aquifer system in which the well was observed before the collapse has changed because of the water level in the deep well of Lu15 well on the original curve. Nakai fitting model is adopted to calculate the earth tide response amplitude factor ratio based on the water level data of Lu15 well, and the possibility of collapse caused by stress change is discussed in combination with non-natural seismic events in Taozhuang Coal mine
\end{abstract}

\section{Introduction}

On the basis of systematically collecting and sorting out the data of the influence of mine earthquakes on the water level dynamics of Lu15 well since 1980 in Taozhuang Coal mine,. CAI Ying-tai et al. [1] studied the characteristics of the co-seismic effect of deep well water level, discovered the precursory phenomenon of mine earthquakes at deep well water level, and discussed the physical mechanism of the effect of mine earthquakes at exploration well water level. Ma Zhifeng et al. [2], based on the analysis of the geological structure and the distribution characteristics of ore earthquakes in Taozhuang coal mine, discussed the occurrence conditions and mechanism of ore earthquakes, put forward the correlation between ore earthquakes and natural earthquakes, and clarified the precursor characteristics of ore earthquakes and their prevention measures. Mine shock is an earthquake phenomenon caused by underground mining. Because its formation process and the natural earthquake have some similarities, some scholars at home and abroad put forward. Natural earthquake prediction should be explored using mine earthquake monitoring [1]. In order to verify the possibility of this hypothesis, dynamic data of lu15 well water level and collapse data of Taozhuang Coal Mine from 2012 to the present were collected in this paper to discuss the response mechanism of Lu15 well water level to mine collapse. Then, by using Nakai fitting model and water level data to calculate the amplitude factor ratio of solid tide response to volume strain, the strain state in the crust was preliminarily judged, and the process of stress accumulation and release was discussed in combination with meteorological elements and collapse data to obtain certain understanding and verification.

\section{Observation well and General situation of Taozhuang Coal Mine}

Well LU15 is located in the Taozhuang Coal Mine area in the west of Zaozhuang Coal Mine in Shandong province, in the south of Beishan Fault.This well was drilled by the geological exploration team of Zaozhuang Coal Mining Bureau in 1977, and drilled to a depth of $501.61 \mathrm{~m}$ and a diameter of $108 \mathrm{~mm}$ under casing to a depth of $239.63 \mathrm{~m}$. The aquifer of Lul5 well is Permian sandstone, buried $239.63 \mathrm{~m}$ deep at the top of the aquifer, and exposed $181.37 \mathrm{~m}$ thick. The groundwater type is pore fissure confined water, and the buried depth of well water is about $44 \mathrm{~m}$. since 2012 , the digital seismic network center, as they passed through Shandong province using minute value digital instruments, and is equipped with three elements of meteorological instruments installation, all instrument observation Wells good operations; The annual variation trend of water level is clear. Affected by rainfall, the water level generally rises from May to August every year, falls from September to October, and is relatively stable from November to May of the next year. The annual variation range of water level is 1.0 to $1.5 \mathrm{~m}$. The well has a strong ability to show crustal stress changes. The diurnal difference of the water level is 12$17 \mathrm{~cm}$, and the well water pressure effect is $3.1 \mathrm{~mm} / \mathrm{hPa}$.(fig1 )It can record some distant seismic waves[3].

\footnotetext{
*LIN Mei:
} 


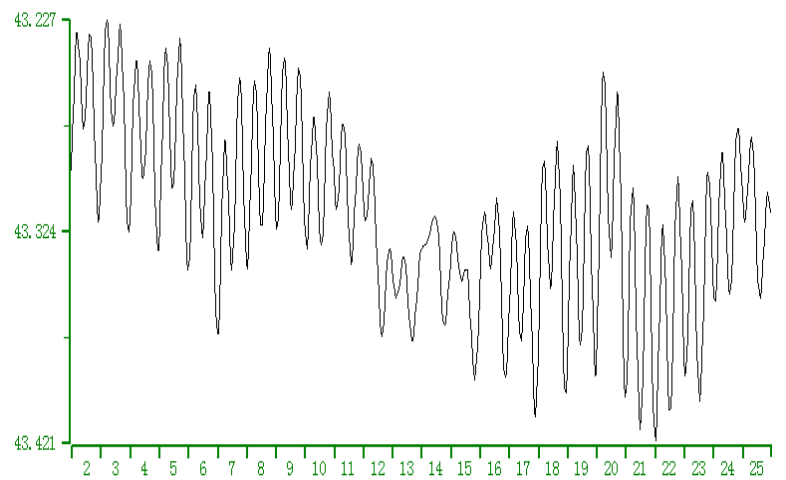

Fig. 1. Dynamic changes of water level in Zaozhuang Lu. 15 well from March 2 to 25, 2019.

The coal seams of the Taozhuang Coal mine lie just below the aquifer, about $600-900 \mathrm{~m}$ above the ground. Because the confined aquifer is sensitive to the stress and strain it receives, the collapse of Taozhuang coal mine also significantly reflects the water level of Lul5 well. The water level in Lu15 well has obvious solid scene effect and air pressure effect. The water level curve in FIG. 1 is the water level curve after removing the annual change trend. With economic development and resource depletion, the production capacity of Taozhuang Coal Mine has decreased in recent years, and it was completely stopped in January 2016. However, due to excessive mining in the mine area, the geological structure is relatively complex, and the collapse occurs from time to time..

\section{Response of lu15 well water level to mine collapse}

If a well aquifer system is thought of as a horizontal layered artesian aquifer system, the variation of well head under vertical stress should satisfy the following equation

$$
\Delta \mathrm{H}=-\frac{n \beta+(1-n) a}{2 \gamma \beta} * \Delta \sigma_{z}
$$

Where $\mathrm{n}$ is the porosity of the table water layer, $\beta$ is the volume compression coefficient of the water in the aquifer, and $\mathrm{a}$ is the volume compression of the solid skeleton of the aquifer Coefficient, $\gamma$ is the specific gravity of the water in the aquifer, $\sigma$ is the vertical stress on the aquifer. It can be seen from Equation (1) that the change of well head is related to the parameters of the aquifer. When the parameters remain unchanged, it is directly proportional to the change of vertical stress received by the aquifer. The coal seam of Taozhuang Coal mine is under the aquifer of Well L5, about 400m away from the aquifer. After the coal is mined out, if the rock layer in the mining area is relatively weak or broken, the rock block will collapse while mining the coal, which will not cause the collapse of the coal mine earthquake. However, if the rock strata are relatively complete, the coal seam does not collapse immediately after mining, but to be suspended large area of rock collapse, resulting in the collapse of the coal mine earthquake. Since the mine collapse occurs below the aquifer, the impact of the collapse creates an impact-downward tension on the aquifer, causing a sudden and rapid drop in the well water level (FIG. 2). The greater the impact force generated during the mine collapse, the greater the downward tension exerted on the aquifer bottom surface, the greater the impact strain generated by the aquifer, and the greater the corresponding change in the water level of the confined well. According to the record of lu15 well water level, the largest collapse produced changes as much as $456 \mathrm{~mm}$. At $15: 14$ on March 9, 2020, it was $12 \mathrm{~km}$ away from the Taozhuang earthquake station and $5 \mathrm{~km}$ away from well $15, \mathrm{Ms}=0.4$ collapse, as shown in the figure 3 :

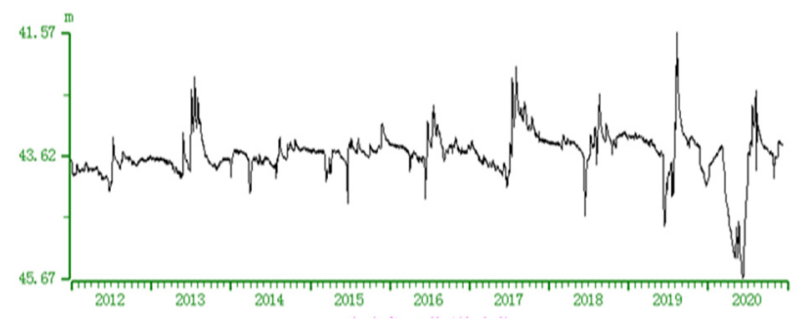

The water leve in Zaozhuang Lu. 15 well

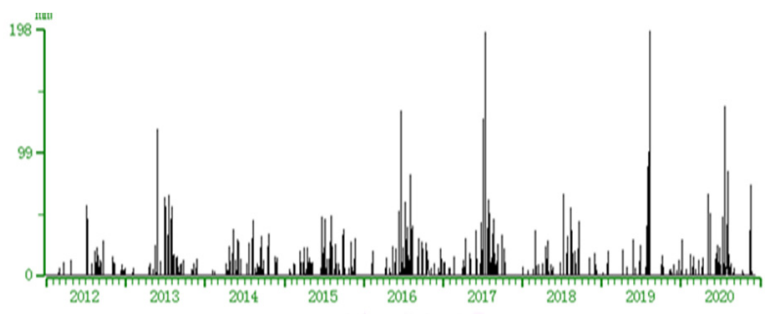

The rainfall in Zaozhuang Lu. 15 well

Fig. 2. Dynamic curve of water level and rainfall in Zaozhuang. Lu 15 well from 2012 to 2020.

According to the above theory, combined with 2012 - December 2020, water level and rainfall contrast curve, we found that the trend of lu 15 Wells in May - June each year Ms 0.1-Ms0.6 tao chong coal mine collapse, causing water brunt, dynamic curve downward, tao chong coal mine in the bottom aquifer, along with increased rainfall, aquifer under the action of rainfall and rock mass unloading rebound, generate tension along the strata within certain limits, and along the vertical rock joints, and other random fracture surface cracking, linking up until the formation of sliding collapse.

\section{Calculation method and result analysis of amplitude factor ratio}

\subsection{Calculation method}

It has been proved by the predecessors that the tidal phenomenon of deep well water level is the response to earth tide, and the feasibility of using the tide of underground water level to calculate the loading and unloading response ratio of earth tide is demonstrated. 
Combined with the calculation and analysis of several typical seismic examples, it is considered that the ratio of amplitude factor of response before earthquake increases. Therefore, the amplitude factor ratio of well water level to crustal tidal strain response can be used to preliminarily judge the strain state in the crust. LAN Shuangshuang et al[4]. Believe that morphologically, the tidal loading and unloading response at the well water level under normal conditions is lower and stable than the background value, and fluctuates up and down with "1" as the mean line. When the seismogenic system is in an unstable state, the response is usually higher than the abnormal change in the calculation value, or there is a large jump, continuous jump phenomenon.

The typical model of horizontal layered confined aquifer is adopted, and it is assumed that all the aquifers are well sealed except the wellhead. Then, under the condition of no drainage, according to the theory of pore elastic medium, the load acting on a plane of the aquifer is shared by solid particles and pore fluids between particles respectively, thus the relationship between well water level change and volume strain can be deduced.

$$
\left.d h=\frac{\theta}{\rho g\left[(1-n) E_{m}+n E_{W}\right]}\right]
$$

Where $\mathrm{dh}$ is the change of water head in aquifer, $\theta$ is the stress variable of aquifer body, $\rho$ is the density of water, $\mathrm{g}$ is the acceleration of gravity, $\mathrm{n}$ is the porosity of aquifer medium, Em is the volume modulus of solid rock particles in aquifer, and Ew is the volume modulus of water. When the above parameters of the well aquifer system are determined unchanged, the change of well water level is proportional to that of the system.

In practice, the amplitude factor ratio of observed ground water level earth tide value to volume strain earth tide response can be obtained by approximating Nakai fitting model:

$$
\mathrm{H}_{\theta}(t)=\left\{\begin{array}{l}
a^{+} R(t)-\beta^{+} \frac{d R}{d t}+K_{0}^{+}+K_{1}^{+} t+K_{1}^{+} t^{2} \\
a^{-} R(t)-\beta^{+} \frac{d R}{d t}+K_{0}^{-}+K_{1}^{-} t+K_{1}^{-} t^{2}
\end{array}\right.
$$

Type, $H \theta(t)$ for the actual observations of ground water level, a for the fitting of a tidal factor, $\mathrm{R}(\mathrm{t})$ for $\mathrm{t}$ time body strain earth tide theory, the $\beta$ is associated with the phase lag of tidal observation coefficient, $\mathrm{K} 0, \mathrm{~K} 1, \mathrm{~K} 2$, respectively of tidal constants (instrument zero drift), speed and acceleration coefficient, using the least squares method to calculate the values of the parameters in the type. [5]

\subsection{Result analysis of amplitude factor ratio}

In this paper, $\mathrm{N}=24$ (that is, the hourly value data of a day is a group) is selected to calculate the amplitude response ratio based on the ground water level earth tide observation data of Well Lu15. Most of the normal values of the calculated amplitude-factor response ratio of the observation Wells fluctuated roughly around 1, as shown in FIG. 3. Only a few points exceeded 1 times the mean square error control line.

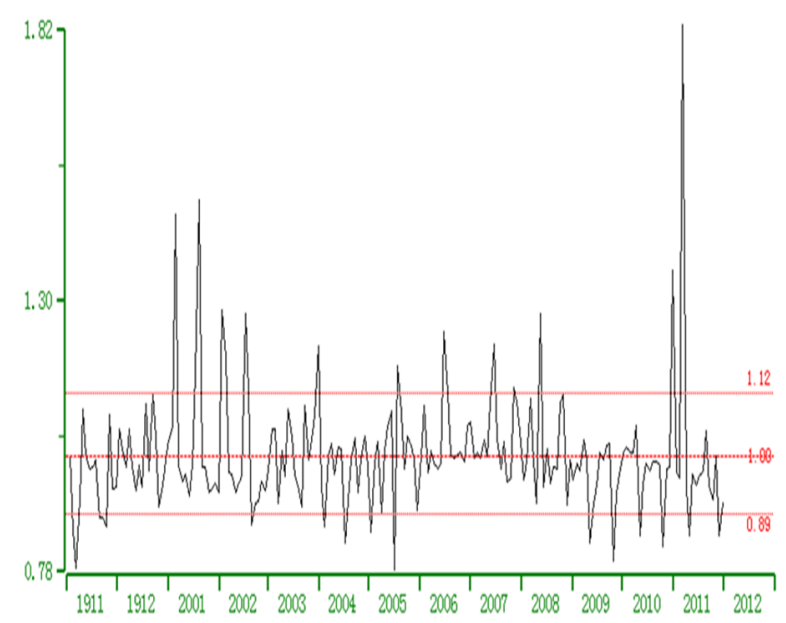

Fig. 3. The amplitude factor response ratio of water level earth tide observation

In order to increase the earth tide information, first of all to preliminary data (processing), the paper calculated the amplitude of the observation data of the tidal level factor response ratio, and draw in November 2019 November 2020 data in the Fig3. The response ratio of more than 1 time of mean square error was selected as the anomaly determination index, and the normal value fluctuated around 1 basically. At 15:14 on March 9, 2020, $12 \mathrm{~km}$ away from the Taozhuang earthquake station and $5 \mathrm{~km}$ away from well 15 , a Ms0.4 landslide occurred. In recent ten years, the biggest sudden drop of water level reached as much as $456 \mathrm{~mm}$, and the decline lasted for a long time as shown in Fig 2. According to the calculated value of the response ratio of amplitude factor, it was found that the response ratio in January and February 2020 increased in the Fig3, with a higher frequency of over 1 times of mean square deviation. After the collapse of Ms0.4 in Taozhuang Coal mine on March 6,2020, the response ratio tended to be flat. It shows that the change of the amplitude-factor ratio of the water level to the volumetric strain of the earth tide is a reflection of the stress change in the crustal water-bearing medium. Before the collapse of Taozhuang Coal mine, due to the accumulation of stress, the amplitude-factor ratio would have a high value change. On October 28, 2020, there was a 0.16-stage blasting, with a small abrupt drop in water level and a high response ratio at the same time. Due to the shallow geological structure changes caused by blasting, the stress increased rapidly and returned to normal after 10 days.

\section{Discussion}

Because the mine collapse occurs below the aquifer, the impact of the collapse creates an impact-downward tension in the aquifer, causing a sudden and rapid drop in the well water level. The change of water level in the collapse response of lu15 well is related to the strain of the aquifer, and the strain of the aquifer is related to the impact force generated by the collapse of the mine. 
Therefore, the response of Lu15 well to the collapse of the mine reflects the strain of the aquifer.

By comparing and analyzing the dynamic data of rainfall and water level, it is found that there is a good correspondence between the annual increase of rainfall and the downward abrupt curve of water level collapse response. By calculating and analyzing the response ratio of amplitude factor, it is found that the process of rainfall is also a process of loading and unloading for underground media. When the aquifer is under the action of rain water and rock unloading rebound, a tension action occurs along the rock strata within a certain range, and it gradually cracks along the vertical joints and other random fracture surfaces of the rock strata, and passes through upward until sliding collapse is formed, which may cause the collapse of ms0.3-MS0.6 or so to occur in the annual rainfall season.

By comparing the landslide parameters of Taozhuang Coal mine in 2020 with the calculation results of the response ratio of the amplitude factor before and after the earthquake in the observation well of water level and earth tide in Lu15 well, it is found that there is a significantly high value anomaly in The first 1-2 months before the response ratio of the water level amplitude factor before $\mathrm{Ms} \geq 0.3$ in the range of $20 \mathrm{~km}$ in the structure of Taozhuang Coal mine. There are obvious relative changes of stress high value response to surface blasting earthquake. It is proved that the amplitude factor response ratio of well water level earth tide observation is an effective method to identify and extract earthquake precursors.

\section{References}

1. CAI Ying-tai et al.(1995) Ore shock effect of deep well water level Acta Seismologica Sinica 1(17):8995

2. Ma Zhifeng et al. (2002) Earthquake disaster and prevention in Taozhuang Coal Mine URNAL OF CATASTROPHOI OGY 4(17):60-63

3. Meng XiaohuaJ . (2011) Seismic information of deep well water level in Taozhuang mining area $J$ Of Institute of Disaster Prevention Seismic information 3 (13) :51-54

4. LAN Shuangshuang, CHI Baoming.(2011) Anomaly analysis of solid tide loading and unloading response ratio of groundwater level before Wenchuan Earthquake Journal of Seismological Research 34(3), 110-116

5. Zhang Zhaodong, Chen Xuezhong,(1997) Chen Jianmin. Seismic precursors of tidal loading and unloading response ratio at well water level Acta Seismologica Sinica 1997, 19(2): 174-180 